\title{
Performance Analysis of Two-Way AF Cooperative Networks with the Nth Relay Selection
}

\author{
Yao Chenhong ${ }^{12 *}$, Pei Changxing ${ }^{1}$ and Guo Jing ${ }^{3}$ \\ ${ }^{I}$ State Key Laboratory of Integrated Services Networks, Xidian University, Xi'an, \\ 710071, Shaanxi Province, China \\ ${ }^{2}$ Xian University of Architecture and Technology, Xi'an 710055, Shaanxi \\ Province, China \\ ${ }^{3}$ Shanxi University of Science \& Technology Xi'an 710021, Shaanxi Province, \\ China \\ E-mail: xiaoyaoff@163.com
}

\begin{abstract}
In this letter, we propose a novel two-way amplify-and-forward $(A F)$ relay selection scheme over independent flat Nakagami-m fading channels. In the proposed schemes, two users first sequentially broadcast their respective information to all the relays. Then, the Nth best-relay perform AF protocol on the received signals sent from the two users and forward them to both users. The Nth best-relay is selected to optimize the end-to- end performance of the system transmission. The performance of the proposed system is quantified by deriving the lower bound of the overall outage probability and average symbol error rate (SER). The effectiveness of our proposed selection scheme and analytical results is verified by simulations.
\end{abstract}

Keywords: Two-way relaying network, amplify-and- forward (AF), outage probability, symbol error rate.

\section{Introduction}

Two-way relaying system has attracted much research interest because of its potential in achieving higher spectral efficiency and throughput for wireless networks [1]. Based on different processing at the relay, such as decode-and-forward and amplify-and-forward, achievable rate regions for various two-way relaying systems have been studied in [2-3]. In [4], the authors investigated the performance of two-way amplify-and-forward relaying networks over independently but not necessarily identically distributed Nakagami-m fading channels in terms of outage probability, average SER, and average sum-rate. Adaptive two-way relaying, which the relay $\mathrm{R}$ adaptively selects AF or DF depending on the decodability of the two bi-directional data streams received at $\mathrm{R}$, has been introduced in [5]. Because of synchronization and a lot of messages exchanging among different relays, a proper relay selection scheme will play a key role in the multiple relay system performance. In [6], the authors derived a simple two-way amplify-and-forward relay selection criterion for the general case over Nakagami-m fading channels. In [7], the authors proposed the max-min sum rate selection algorithm for AF bidirectional network based on the outage probability. Twoway relay selection, which is introduced for differential modulation systems to improve system performance, has been proposed in [8].

One of the key features in the two-way relaying networks is that the two-way protocol should not only consider the downlink (broadcasting) but also the uplink (multiple-access) data flows in the same time, which can improve the overall performance of the two directional transmission. However, most of the recent research 
foucs on the "best" single relay selection methods. In practice, the best relay may not always be selected, thus the study of the $N$ th best relay selection will be very necessary.

In [9], the outage performance of the proposed relay selection scheme is analyzed under the outdated channel state information (CSI), and a tight closed-form lower bound and asymptotic value of the outage probability over Rayleigh fading channels are obtained. Analysis of amplify-and-forward with the Nth best-relay cooperative diversity over Rayleigh fading channels is presented in [10]. The authors consider the adaptive DF and AF cooperative diversity systems with the Nth best-relay selection scheme in [11]. In [12], authors assume that the relay employs the amplify-and-forward protocol, propose the Nth worst relay selection scheme and analyze the system performance of the system under two different fading models: lognormal shadowing channels and generalized-K fading channels. In [13], the asymptotic symbol error rate (SER) and diversity gain of AF cooperative communications with the Nth best-relay scheme over independent and nonidentical Nakagami-m fading channels are derived. The authors investigate the performance of Nth best relay selection networks with secrecy constraints where several eavesdroppers try to overhear the source message in [14].

Motivated by all of the above, this letter presents a novel two-way relay selection scheme and we investigate the performance of the proposed system over independent flat Nakagami-m fading channels. Firstly, the lower bound of the overall outage probability for the proposed system is investigated. And then, the average symbol error rate (SER) are determined. Finally, Simulation results verify our analysis.

\section{System Model}

Consider a two-way amplify-and-forward (AF) relaying system, where two sources $S_{1}$ and $S_{2}$ exchange information with the help of $K$ cooperating AF relay nodes $k, k \in\{1, \ldots, K\}$, over independent flat Nakagami-m fading channels. We assume that all terminals are single-antenna devices and operate in a half-duplex mode, and there is no direct path between $S_{1}$ and $S_{2}$. We also assume here the additive white Gaussian noise (AWGN) at all nodes is independent and identically distributed (i.i.d.) $C \mathcal{N}\left(0, N_{0}\right)$.

In the first time slot, $S_{1}$ and $S_{2}$ transmit their signals $x_{1}$ and $x_{2}$ to all the relays simultaneously. So the received signal at relay $k$ can be expressed as

$$
y_{k}=\sqrt{P_{1}} h_{1 k} x_{1}+\sqrt{P_{2}} h_{2 k} x_{2}+\eta_{k},
$$

where $P_{1}$ and $P_{2}$ are the transmitting power of $S_{1}$ and $S_{2}$. $h_{1 k}$ and $h_{2 k}$ stand for the channel gains of $S_{1} \rightarrow k$ and $S_{2} \rightarrow k$, respectively.

In the next two time slots, the $N$ th relays $R_{k}$ is selected for amplifying its received signal and forwarding it. Thus, in the second time slot, Considering the channel is block time variation, the received signal at relay $k$ is amplified with the fixed-gain [17], $G=\sqrt{1 / C N_{0}}$, whereby $C$ is a constant and retransmitted to $S_{1}$ and $S_{2}$, which can be expressed by

$$
\begin{aligned}
& y_{s 1}=G \sqrt{P_{1}} g_{1 k}^{\dagger} h_{1 k} x_{1}+\sqrt{P_{2}} g_{2 k}^{\dagger} h_{2 k} x_{2}+g_{1 k}^{\dagger} \eta_{k}+\eta_{1}, \\
& y_{s 2}=G \sqrt{P_{1}} g_{2 k}^{\dagger} h_{1 k} x_{1}+\sqrt{P_{2}} g_{2 k}^{\dagger} h_{2 k} x_{2}+g_{2 k}^{\dagger} \eta_{k}+\eta_{2},
\end{aligned}
$$

respectively. where $g_{1 k}^{\dagger}, g_{2 k}^{\dagger}$ are the conjugates of $g_{1 k}$ and $g_{2 k}$.

After the self-interference parts are subtracted [1], the received SNRs of twolinks $S_{2} \rightarrow k \rightarrow S_{1}$ and $S_{1} \rightarrow k \rightarrow S_{2}$ are given by

$$
\gamma_{1 k}=\frac{P_{2} \gamma_{g_{1 k}} \gamma_{h_{2 k}}}{\gamma_{g_{1 k}}+C}
$$




$$
\gamma_{2 k}=\frac{P_{1} \gamma_{g_{2 k}} \gamma_{h_{1 k}}}{\gamma_{g_{2 k}}+C},
$$

respectively, where $\gamma_{g_{s k}}=\left|g_{s k}\right|^{2} / N_{0}, \quad, \gamma_{h_{s k}}=\left|h_{s k}\right|^{2} / N_{0}, \quad s=1,2$ and $g_{1 k}$ and $g_{2 k}$ stand for the channel gains of $k \rightarrow S_{1}$ and $k \rightarrow S_{2}$, respectively.

Since the overall performance of the considered system is governed by the performance of the weakest source, let $\gamma_{k}=\min \left(\gamma_{1 k}, \gamma_{2 k}\right)$. Thus, the relay selection criterion can be expressed as

$$
\left(k^{*}\right)=\arg \mathrm{N}_{t h} \max _{k \in\{1, \cdots, N\}}\left\{\gamma_{k}\right\},
$$

Where $\mathrm{N}_{t h} \max _{k \in \mathcal{C}\{\cdot\}}$ denotes to select the $N$ th maximum value from the set $C$.

\section{Performance Analysis}

In this section, performance of the above-mentioned relay selection schemes under Nakagami-m is analyzed. First of all, closed-form lower bound for the overall outage probability is derived. Then, useful insights into practical systems implementation are obtained by quantifying SER.

\subsection{Outage Probability}

With the above selection scheduling, the overall outage probability is given by

$$
P_{\text {out }}=P_{r}\left[\mathrm{~N}_{t h} \max _{k \in\{1, \cdots, \mathrm{K}\}}\left\{\gamma_{k}\right\} \leq \gamma_{0}\right] \text {. }
$$

Since that is hard to obtain the exact result of (7), we derive the tight lower bound of the outage probability. First, from (2) and (3), we can obtain

$$
\begin{gathered}
\gamma_{1 k}=\frac{G^{2}\left|g_{1 k}\right|^{2}\left|h_{2 k}\right|^{2} \gamma_{2}}{G^{2}\left|g_{1 k}\right|^{2}+1}<\gamma_{2}\left|h_{2 k}\right|^{2}, \\
\gamma_{2 k}=\frac{G^{2}\left|g_{2 k}\right|^{2}\left|h_{1 k}\right|^{2} \gamma_{1}}{G^{2}\left|g_{2 k}\right|^{2}+1}<\gamma_{1}\left|h_{1 k}\right|^{2} .
\end{gathered}
$$

Then, the overall outage probability is given by

$$
\begin{gathered}
P_{\text {out }}>P_{\text {out }}^{l b} \\
=F_{r_{k} *}\left(\gamma_{0}\right) \\
=\operatorname{Pr}\left[\mathrm{N}_{t h} \max _{k \in\{1, \cdots, K\}} \min \left\{\gamma_{1 k}, \gamma_{2 k}\right\} \leq \gamma_{0}\right]
\end{gathered}
$$

where $F_{\gamma_{k}}(x)=\operatorname{Pr}\left[\min \left(\gamma_{1 k}, \gamma_{2 k}\right) \leq x\right]$ is the $\mathrm{CDF}$ of $\gamma_{k}$.

Under Nakagami-m fading, $\left|h_{1 k}\right|^{2}$ and $\left|h_{2 k}\right|^{2}$ for any $k \in\{1, \cdots, N\}$ are independent but not necessarily identically distributed with shape parameter $m_{1 k}, m_{2 k}$ and scale parameter $\Omega_{1 k}=E\left[\left|h_{1 k}\right|^{2}\right], \Omega_{2 k}=E\left[\left|h_{2 k}\right|^{2}\right]$ denotes the expectation operator. Hence, the cumulative distribution function (CDF) of the fading amplitude $\left|h_{s k}\right|^{2}, \mathrm{~s}=1,2$, can be given by

$$
F_{h_{s k}}\left(x_{s}\right)=1-\frac{\Gamma\left(m_{s k}, \frac{m_{s k}}{\Omega_{s k}} x_{s}\right)}{\Gamma\left(m_{s k}\right)},
$$

where $\Gamma(\cdot)$ is the gamma function, $\Gamma(\cdot, \cdot)$ is the incomplete gamma function [19].

The CDF for $\gamma_{i}$ can be expressed by 


$$
\begin{gathered}
F_{\gamma_{k}}(\mathrm{x})=\operatorname{Pr}\left[\min \left\{\gamma_{1 k}, \gamma_{2 k}\right\} \leq x\right] \\
=F_{h_{1 k}}(x)+F_{h_{2 k}}(x)-F_{h_{1 k}}(x) F_{h_{2 k}}(x) \\
=\frac{\Gamma\left(\mathrm{m}_{1 k}, \frac{m_{1 k}}{\Omega_{1 k}} x\right)}{\Gamma\left(\mathrm{m}_{1 k}\right)}+\frac{\Gamma\left(\mathrm{m}_{2 k}, \frac{m_{2 k}}{\Omega_{2 k}} x\right)}{\Gamma\left(\mathrm{m}_{2 k}\right)} \quad-\frac{\Gamma\left(m_{1 k}, \frac{m_{1 k}}{\Omega_{1 k}} x\right)}{\Gamma\left(m_{1 k}\right)} \frac{\Gamma\left(m_{2 k}, \frac{m_{2 k}}{\Omega_{2 k}} x\right)}{\Gamma\left(m_{2 k}\right)}
\end{gathered}
$$

By substituting (12) into (10), the desired result of the lower bound of the overall outage probability $P_{\text {out }}^{l b}$ can be derived as in (13).

$$
\begin{aligned}
& P_{\text {out }}^{l b}=\sum_{k=1}^{K}\left(\begin{array}{l}
K \\
k
\end{array}\right)\left[\frac{\Gamma\left(m_{1 k}, \frac{m_{1 k}}{\Omega_{1 k}} \gamma_{0}\right)}{\Gamma\left(m_{1 k}\right)}+\frac{\Gamma\left(m_{2 k}, \frac{m_{2 k}}{\Omega_{2 k}} \gamma_{0}\right)}{\Gamma\left(m_{2 k}\right)}-\frac{\Gamma\left(m_{1 k}, \frac{m_{1 k}}{\Omega_{1 k}} \gamma_{0}\right)}{\Gamma\left(m_{1 k}\right)} \frac{\Gamma\left(m_{2 k}, \frac{m_{2 k}}{\Omega_{2 k}} \gamma_{0}\right)}{\Gamma\left(m_{2 k}\right)}\right]^{k} \\
& \left(1-\frac{\Gamma\left(m_{1 k}, \frac{m_{1 k}}{\Omega_{1 k}} \gamma_{0}\right)}{\Gamma\left(m_{1 k}\right)}\right)^{K-k}\left(1-\frac{\Gamma\left(m_{2 k}, \frac{m_{2 k}}{\Omega_{2 k}} \gamma_{0}\right)}{\Gamma\left(m_{2 k}\right)}\right)^{K-k} \\
& =\sum_{k=N}^{K}\left(\begin{array}{l}
K \\
k
\end{array}\right) \sum_{p=1}^{k}\left(\begin{array}{l}
k \\
p
\end{array}\right)(-1)^{p}\left(1-\frac{\Gamma\left(m_{1 k}, \frac{m_{1 k}}{\Omega_{1 k}} \gamma_{0}\right)}{\Gamma\left(m_{1 k}\right)}\right)^{K-k+p}\left(1-\frac{\Gamma\left(m_{2 k}, \frac{m_{2 k}}{\Omega_{2 k}} \gamma_{0}\right)}{\Gamma\left(m_{2 k}\right)}\right)^{K-k+p}
\end{aligned}
$$

\subsection{SER analysis}

We invoke the cumulative distribution function (CDF) approach described in [18] and derive the exact SER in terms of the CDF of $\gamma_{k^{*}}, F_{\gamma_{k^{*}}}(x)$, as

$$
P_{e}=\frac{a}{2} \sqrt{\frac{b}{\pi}} \int_{0}^{\infty} \gamma^{-\frac{1}{2}} \exp (-\mathrm{b} \gamma) F_{r_{k^{*}}}(\gamma) \mathrm{d} \gamma
$$

This approach embraces all general modulation schemes that have an SER expression of the form $P_{e}=E\{Q(\sqrt{c \gamma})\}$, where a and $\mathrm{b}$ are modulation specific constants. The modulation formats to which (14) apply include binary phase- shift keying (BPSK) $(\mathrm{a}=1, \mathrm{~b}=1)$, quadrature phase-shift keying (QPSK) $(\mathrm{a}=1, \mathrm{~b}=0.5)$, and $\mathrm{M}$-ary pulse amplitude modulation (M-PAM) $\left(\mathrm{a}=2(\mathrm{M}-1) / \mathrm{M}, \mathrm{b}=3 /\left(\mathrm{M}^{2}-1\right)\right)$.

Then, Inserting (13) into (14), we evaluate the BER performance evaluate the BER of binary phase shift keying (BPSK), the SER can be expressed as

$$
P_{e}=\frac{1}{2} \sqrt{\frac{1}{\pi}} \int_{0}^{\infty} \sum_{k=N}^{K}\left(\begin{array}{l}
K \\
k
\end{array}\right) \sum_{p=1}^{k}\left(\begin{array}{l}
k \\
p
\end{array}\right)(-1)^{p} \gamma^{-\frac{1}{2}} \exp (-\mathrm{b} \gamma)
$$




$$
\left(1-\frac{\Gamma\left(m_{1 k}, \frac{m_{1 k}}{\Omega_{1 k}} \gamma_{0}\right)}{\Gamma\left(m_{1 k}\right)}\right)^{K-k+p}\left(1-\frac{\Gamma\left(m_{2 k}, \frac{m_{2 k}}{\Omega_{2 k}} \gamma_{0}\right)}{\Gamma\left(m_{2 k}\right)}\right)^{K-k+p} d \gamma
$$

Unfortunately, there is no closed-form expression for the integral in (15).

Under the assumption of linear modulation and AWGN, the SER conditioned on the instantaneous received SNR is approximately $Q(\sqrt{c \gamma})$, where c is a constant determined by the modulation format (e.g. $\mathrm{c}=2$ for phase shift keying (PSK)), and $Q(x)=\frac{1}{2 \pi} \int_{x}^{\infty} \exp \left(-\mathrm{t}^{2} / 2\right) d t$.The average SER is therefore

$$
P_{e}=E\{Q(\sqrt{c \gamma})\},
$$

where $\mathrm{E}(\cdot)$ denotes the expectation operator. This integral can be computed by using the Gauss-Hermite integration as (17), where $\mathrm{M}$ denotes the number of points for the Gauss-Hermite integration, $x_{n}$ and $\omega\left(x_{n}\right)$ stands for the abscissas and weights of GaussHermite integration, respectively.

$$
\begin{gathered}
P_{e}=\frac{1}{2 \sqrt{\pi}} \sum_{k=N}^{K}\left(\begin{array}{l}
K \\
k
\end{array}\right) \sum_{p=1}^{k}\left(\begin{array}{l}
k \\
p
\end{array}\right)(-1)^{p} \sum_{n}^{M} \omega\left(x_{n}\right) \\
\left(1-\frac{\Gamma\left(m_{1 k}, \frac{m_{1 k}}{\Omega_{1 k}} \gamma_{0}\right)}{\Gamma\left(m_{1 k}\right)}\right)^{K-k+p}\left(1-\frac{\Gamma\left(m_{2 k}, \frac{m_{2 k}}{\Omega_{2 k}} \gamma_{0}\right)}{\Gamma\left(m_{2 k}\right)}\right)^{K-k+p}
\end{gathered}
$$

\section{Numerical Results}

In this section, we present Monte-Carlo simulations to confirm the derived analytical results. We assume that the average SNR in links from two sources to relay is equal, i.e. $P_{1}=P_{2}$. The distance between two sources $S_{1}$ and $S_{2}$ is normalized to 1 , the normalized distance from $S_{1}$ to relay $k(k=1, \cdots, K)$ is $d_{1 k}$, and the path loss exponent is 3 . Thus, $\Omega_{1 k}=d_{1 k}^{-3}, \Omega_{2 k}=\left(1-d_{1 k}\right)^{-3}$. Without loss of generality, we set $d_{1 k}=0.5$ for all the relays. Furthermore, we assume $m_{1 k}=0.5, m_{2 k}=0.5, k=1, \cdots, K$, and the targeted transmission rate $\mathrm{R}=1 \mathrm{bit} / \mathrm{s} / \mathrm{Hz}$.

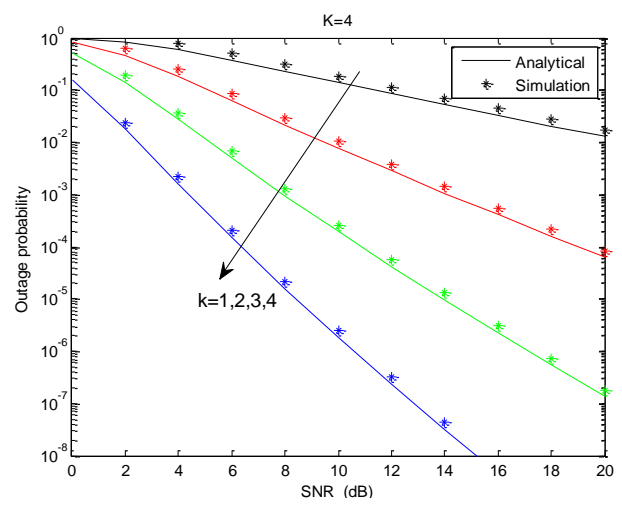

Figure 1. Outage Probability AGAINST SNR for $K=4$ 


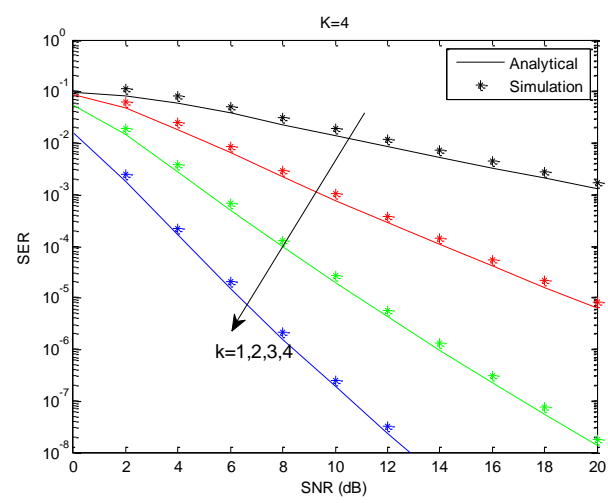

Figure 2. Average Symbol Error Rate against SNR for $K=4$

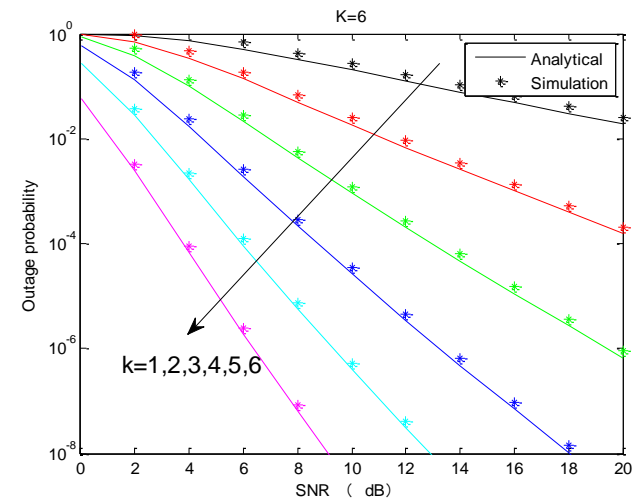

Figure 3. Outage Probability against SNR for $K=6$

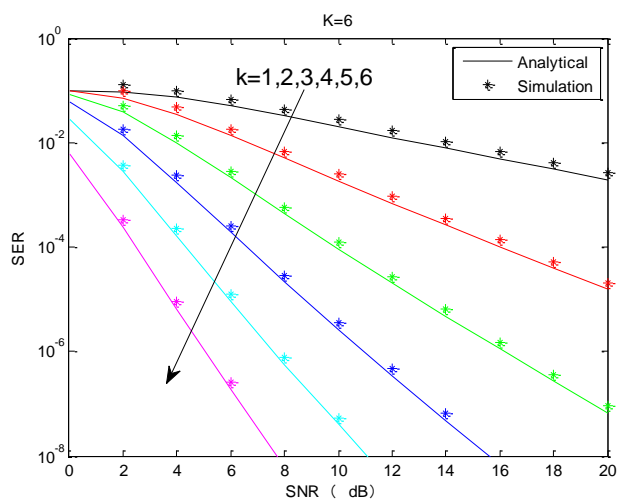

Figure 4. Average Symbol Error Rate against SNR for $K=6$

Figure 1 and Figure 3 shows the theoretical results for the overall outage probability, and actual simulation of the proposed scheme for $K=4$ and $K=6$, respectively. From Figure 1 and Figure 3, it is observed that analytical results exactly coincide with Monte Carlo simulations and therefore verify the accuracy of our analysis. It can be seen that the theoretical analysis is validated in all SNR regimes. As expected, we can see that the number of relay $k$ also affects the difference of the overall outage probability between the proposed scheme and the "best" single relay selection protocol, that is, as $K$ gets bigger, the difference becomes more obvious. It can also be seen that the relay gain given by (10) performs a tight lower bound for (6) for the system outage performance.

Figure 2 and Figure 4 shows the theoretical results for the average symbol error rate against SNR, and actual simulation of the proposed scheme for $K=4$ and $K=6$, 
respectively. From Figure 2 and Figure 4, it is observed that analytical results also exactly coincide with Monte Carlo simulations and therefore verify the accuracy of our analysis. As expected, we can see that the number of relay $k$ also affects the difference of SER, that is, as $K$ gets bigger, the difference becomes more obvious. The selection scheme can greatly improve the overall system SER and diversity performance.

\section{Conclusion}

In this letter, we present the amplify-and-forward relay system with the $N$ th bestrelay cooperative diversity over independent flat Nakagami-m fading channels. Closedform expressions for the probability density functions of the output SNR is obtained. Furthermore, closed-form expressions for the outage probability and SER are determined. Results show that the diversity order increases linearly with the number of relays and decreases linearly with the order of the relay. And then, numerical results verify that the proposed relay selection scheme has better outage performance than that of the single relay selection protocol.

\section{Acknowledgements}

The work was supported by the National Natural Science Foundation of China under Grant $(61072067,61372076)$, and funded by the State Key Laboratory of Integrated Services Networks under the Grant ISN 1001004, and by the National Science and Technology Major Project of China under Grant 2009ZX03007-003.

\section{References}

[1] B. Rankov and A. Wittneben, "Spectral efficient protocols for half-duplex fading relay channels", IEEEJ. Select. Areas Commun, vol. 25, no. 2, (2007), pp. 379-389.

[2] S. J. Kim, P. Mitran, and V. Tarokh, "Performance bounds for bidirectional coded cooperation protocols", IEEE Trans. Inform. Theory, vol. 54, (2008), pp. 5235-5241.

[3] B. Rankov and A. Wittneben, "Achievable rate regions for the two-way relay channel", Proc. IEEE ISIT (2006), pp. 1668-1672.

[4] J. Yang, P. Fan, T. Q. Duong and X. Lei, "Exact Performance of Two-Way AF Relaying in Nakagami-m Fading Environment", IEEE Trans. Wireless Commun, vol. 10, no. 3, (2011), pp. 980987.

[5] Q. Li, S. H. Ting, A. Pandharipande and Y. Han, "Adaptive two-way relaying and outage analysis", IEEE Trans. Wireless Commun, vol. 8, no. 6, (2009), pp. 3288-3299.

[6] E. Y. Li and S. Z. Yang, "Simple relay selection criterion for general two-way opportunistic relaying networks", Electron. Lett, vol. 48, no. 14, (2012), pp. 881-882.

[7] X. Zhang and Y. Gong, "Adaptive poweral location in two-way amplify-and-forward relay networks", Proc. IEEE ICC, (2009), pp. 1-5.

[8] L. Song, Y. Li, H. Guo and B. Jiao, "Differential bidirectional relay selection using analog network coding”, Proceedings of IEEE WCNC, (2010), pp. 1-5.

[9] J. Ou, H. Wu, Q. Wang and Y. Zou, "A Simple Nth Best-Relay Selection Criterion for Opportunistic Two-Way Relay Networks under Outdated Channel State Information”, Ksii Transactions on Internet \& Information Systems, vol. 8, no. 10, (2014), pp. 3409-3422.

[10] S. I. Salama and M. H. Ahmed, "On the Performance of Amplify-and-Forward Cooperative Diversity with the Nth Best-Relay Selection Scheme", IEEE International Conference on Communications (2009), pp. 1-6.

[11] S. S. Ikki and M. H. Ahmed, "On the performance of cooperative-diversity networks with the Nth best-relay selection scheme", IEEE Transactions on Communications, vol. 58, no. 11, (2010), pp. 3062-3069.

[12] L. Yang, K. Qaraqe, E. Serpedin and X. Gao, "Performance Analysis of Two-Way Relaying Networks with the Nth Worst Relay Selection Over Various Fading Channels", IEEE Transactions on Vehicular Technology, vol. 64, no. 7, pp. 3321-3327.

[13] S. I. Chu, "Performance of Amplify-and-Forward Cooperative Communications with the N-th BestRelay Selection Scheme over Nakagami-m Fading Channels", IEEE Communications Letters, vol. 15, no. 2, (2011), pp. 172-174.

[14] X. Wang, H. Zhang, T. Duong, Q. M. Elkashlan and V. N. Q. Bao, "Secure Cooperative Communication with Nth Best Relay Selection", Vehicular Technology Conference (VTC Spring), (2014), pp. 1-5. 
[15] Z. Wang and G. B. Giannakis, "A simple and general parameterization quantifying performance in fading channels", IEEE Trans. Commun, vol. 51, no. 8, (2003), pp. 1389-1398.

[16] L. Zheng and D. N. C. Tse, "Dversity and multiplexing: A fundamental tradeoff in multiple antenna channels", IEEE Trans. Inform. Theory, vol. 49, (2003), pp. 1073-1096.

[17] D. B. da Costa and S. Aissa, "Beamforming in dual-hop fixed gain relaying systems", Proceedings of IEEE Int. Conf. Commun, (2009), pp. 1-5

[18] M. R. McKay, A. L. Grant and I. B. Collings, "Performance analysis of MIMO-MRC in doublecorrelated Rayleigh environments", IEEE Trans. Commun, vol. 55, no. 3, (2007), pp. 497C507

[19] I. S. Gradshteyn and I. M. Ryzhik, "Table of Integrals", Series and Products, 6th edition, Academic Press, New York, (2000).
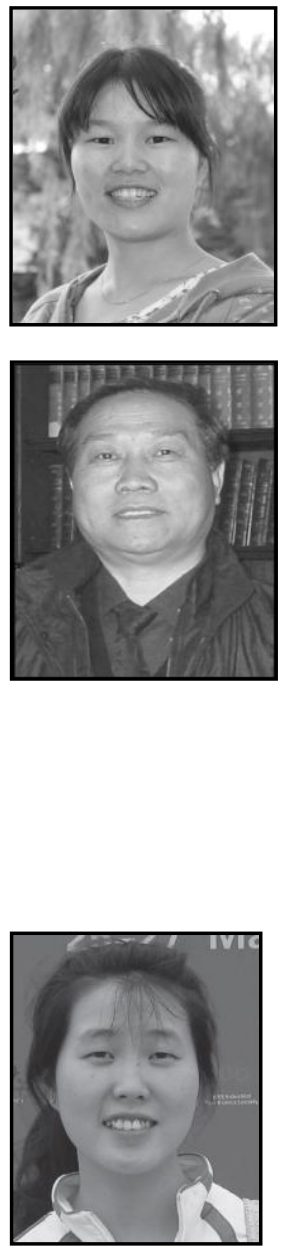

\section{Authors}

Yao Chenhong, she is currently working toward the Ph.D. degree in communication and signal processing with the State Key Laboratory of Integrated Services Networks, Xidian University, Xi' an, China. Her research interests include network measurement, wireless networks, relay networks, and parameter estimation theory. Email: xiaoyaoff@163.com.

Pei Changxing, he is a professor, advisor for doctoral students, senior member of the China Institute of Communication, senior member of China Institute of Electronic, vice-director of the Electronics Institute of Shaanxi Province and the director of Working committee for Academic Exchange. He is mainly engaged in the fields of Radio communication, Anti-jamming communication, Network Measurement, Television system, Highfrequency electronic circuits and Modern filter network. He holds two patents and applied for two. He also has been awarded the prize of scientific and technical accomplishment three times. He has authored eleven technical books. Over hundred papers were published in key journals and academic conferences.

Guo Jing, she received the Ph.D. degree in communication engineering from Xidian University, Xi'an, China. She is currently a lecturer of the institute of Electrical \& Information Engineering, Shaanxi University of Science \& Technology, Xian, Shaanxi, China. Her current research interests include MIMO systems, relayassisted and cooperative communications, and wireless communication theory. 